CORRECTION

\title{
Correction: Policy threats to maternal and child nutrition: putting the unborn child at a lifelong disadvantage
}

Joyce R. Javier ${ }^{1}$ and Vivek Balasubramaniam ${ }^{2}$ on behalf of the Pediatric Policy Council

Pediatric Research (2019) 85:733; https://doi.org/10.1038/s41390-019-0315-x

Correction to: Pediatric Research; 84, 580-581 https://doi.org/ 10.1038/s41390-018-0166-x; Published online: 05 September 2018

Following publication of this article the authors noticed that affiliations were incorrectly assigned. The original article has now been updated so that the author "Joyce R. Javier" is associated with the "Children's Hospital Los Angeles, University of Southern
California Keck School of Medicine, Department of Pediatrics, Division of General Pediatrics, Los Angeles, CA, USA", and the author "Vivek Balasubramaniam" is associated with the "Department of Pediatrics, University of Wisconsin School of Medicine and Public Health, Madison, USA". This has been corrected in both the PDF and HTML versions of the article.

\footnotetext{
${ }^{1}$ Children's Hospital Los Angeles, University of Southern California Keck School of Medicine, Department of Pediatrics, Division of General Pediatrics, Los Angeles, CA, USA and ${ }^{2}$ Department of Pediatrics, University of Wisconsin School of Medicine and Public Health, Madison, USA

Correspondence: Joyce R. Javier (jojavier@chla.usc.edu)
}

Published online: 1 February 2019 